\title{
A Case Study Approach: Iterative Prototyping Model Based Detection of Macular Edema in Retinal OCT Images
}

\author{
Sadaf Sahar, Sadaf Ayaz, M.Usman Akram, Dr. Imran Basit(AFIO) \\ Department of Computer Engineering \\ College of Electrical \& Mechanical Engineering NUST \\ Rawalpindi, Pakistan \\ sadafsahar21@gmail.com, sadafayaz32@gmail.com, usmakram@gmail.com,drimranbasit@gmail.com
}

\begin{abstract}
Highly Reliable Automated medical diagnosis systems are of critical importance. Such systems aid in early detection of diseases and prevention of its further progression. Development of such a reliable and efficient software system is possible using a suitable system development life cycle (SDLC) model only. A SDLC model develops a system in a structured, deliberate and methodical mode and provides a very reliable and efficient system within limited resources and time. Macular edema is the blurring or loss of central vision which is caused as a result of Diabetic Retinopathy and Analysis of OCT images helps in identification of Macular Edema. The aim of this research is the successful detection of Macular Edema using Iterative Prototyping SDLC model. First the extraction of ILM layer has been done by using Active Contour based Segmentation and Curve Fitting Techniques then a new technique is proposed in this research for the successful localization of fovea in retinal ILM layer by using distance based method. Finally the detection of Macular edema has been done on the basis of analysis of fovea region. The system is evaluated using a local dataset of OCT images which is gathered with the help of Armed Forces institute of Ophthalmology. The dataset consists of 550 images and the developed system gives an accuracy of $84 \%$.
\end{abstract}

Keywords-SDLC, Iterative Prototyping, Fovea Centrails, Optical Coherence Tomography, Macula, Macular Edema.

\section{INTRODUCTION}

A System Development Life Cycle (SDLC) is a methodology which is used to represent the process of development of a system and the system is developed in a structured, deliberate and methodical mode which does reiterate every single stage of the Life Cycle. It is the development of a system or an application following the process of planning, creating, testing and deploying for the success of system under development. There are multiple SDLC models like waterfall model, iterative model and incremental model etc. and to ensure the success of system developed, the perfect model to follow is decided. Figure1 shows different phases of a Life Cycle Model.

The most suitable model is selected on the basis of Type of system to be developed, Requirements and Functionalities of the system, Skills and Experience. A suitable SDLC is a key to the success of project.

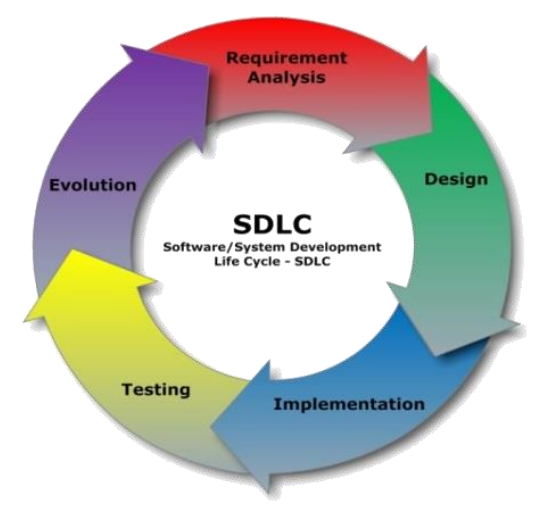

Figure 1: System Development Life Cycle Phases

Rapid prototyping is the type of SDLC model in which quick prototypes are delivered for assessment and after doing modifications, a new prototype is again developed according to new requirements. Edema detection system is aimed to provide highly accurate Edema detection and for that it is necessary to get quick and frequent response from a specialist, during development, who will be using this system. Rapid Prototyping Model is the one which can provide help in development of a system in this way. Hence the development of this system is done using Iterative Prototyping SDLC model.

Macular Edema is the disease in which the macular part of eye gets damaged causing central vision blurring and in severe case the loss of vision. Macular edema is caused due to the leakage of fluids out of blood vessels in retina. The fluids leakage out of blood vessels causes swelling and thickening of Macula and as a result loss of detailed vision. Figure2 (top) shows the macular edema pointed out in an OCT image in form of cystic pockets and rise in Fovea and Figure2 (bottom) shows a fundus image with edema in yellow color. Since Macular Edema causes prominent changes in fovea region in OCT that's why Macular Edema can be identified through the localization of fovea region and after that analysis of fovea region in an OCT image. On the basis of changes occurring in fovea region, Macular Edema can be easily identified. 


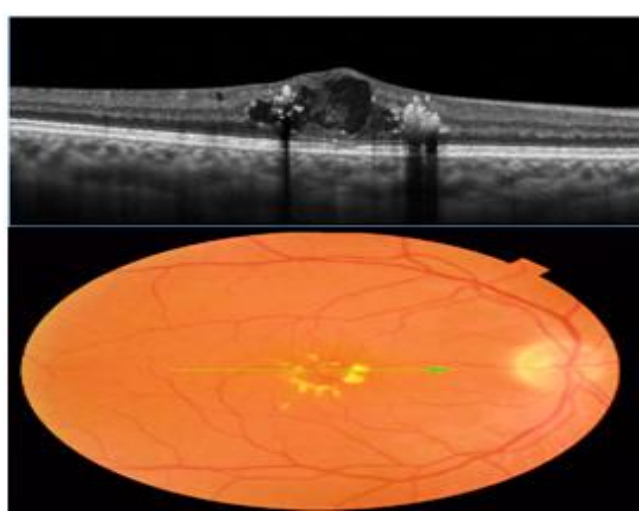

Figure 2: Macular Edema Identification in OCT and Fundus Image

The paper proposes a novel method for identification of Macular Edema in Retinal OCT images using Iterative prototyping SDLC model. Section-I is an introduction of Edema and SDLC model followed by section-II having related work done for Fovea and Edema detection. Section-III is the detailed Methodology section having step by step development of edema detection system. Section-IV describes Results obtained by the system developed and finally Section-V contains conclusion and an overview of the complete system development process.

\section{RELATED WORK}

There is no work done already for Fovea and Edema detection using OCT images but there are many algorithms proposed by many researchers for fovea and edema detection in Digital Fundus Images [13-16]. One is the detection of fovea in fundus images is by using some morphological operation of Image processing. It finds out the location of optic disk first and then identifies the location of fovea. Since fovea is the non-vascular region of fundus image that's why it is the dark most part of the image. In this way a collection of pixels located at the center and dark colored are marked as fovea [1].

Spatial domain filteration is another method which is used for the detection of fovea. Various gray scale image operations are applied on the fundus image first. Then spatial filteration is applied to extract the macular region. After that fovea is identified as the lowest frequency values in the Macula [2].

Since fovea is the non-vascular region of macula and does not have any blood vessels. By using this property of fovea another technique has been proposed for fovea identification. It identifies the presence or absence of fovea on the basis of blood vessels presence or absence. The thickness of blood vessels is calculated in macula and the region with minimum thickness is considered as fovea [3].

Another method for detection of fovea in fundus images is with the help of color bands. A moving window calculates the average color intensity of the image after the extraction of red and green components of fundus image. The window having minimum average intensity value is marked as fovea. These intensities are calculated after the fusion of red and green components [4].
By using the properties of blood vessels and information of optic disk in fundus image, another algorithm based upon optic disk is also proposed [5]. Graphically represented intensities of color bands are also helpful in the detection of optic disk and fovea region [6].

The extraction of retinal layers is also very important before fovea detection in OCT images and there are many methodologies proposed for this. One method is the assigning of normal feature values for all the layers. Then for a given image, scanning is done vertically. When the values of features start to deviate from present values then it is checked in the next layer. If this condition meets then it is marked as the separation mark between the two layers [7].

Probabilistic model is another one for the extraction of the layers. A probabilistic value for each pixel in the layer is calculated after some preprocessing. The value of layer defines the probability of the pixel to belong to that layer. If the value of layer is high then the pixel will more probably belong to that layer. This is done by using random forest classifier which separates it into layers [8]. Table3.1 shows an overview of all the related work done.

Due to tilted OCT images during scanning, it becomes sometimes difficult to process the image easily so the alignment of image is very necessary. The flattering of layers aligns them to the $\mathrm{x}$-axis and then bilinear interpolation can be easily used for image alignment [9] [10]. One method for the detection of Macular Edema in fundus images is through the detection of exudates in fundus images. In this method first of all the detection of Optic Disk is very necessary and it has been done by the use of morphological filteration techniques and watershed transforms. After that exudates have been found by their high variations in their gray levels and morphological re-construction techniques have been used to determine their contours. Finally Macular Edema detection has been done on the basis of exudates presence [11].

In another method for the detection purpose of Macular edema it is necessary to find the presence of all of the possible exudates on retinal surface. In this method the exudates have been enhanced with the help of Gabor filteration bank and a binary mask has been generated for the possible locations of exudates. Hence the Macular edema has been identified on the basis of number and locations of exudates on the surface of retina in colored fundus images [12].

\section{PROPOSED Methodology}

The whole project development is divided into multiple modules and their sub modules. A prototype module is developed for each module and is handed over to a specialist for feedback. The changes mentioned by the specialist are noted and another prototype is developed on the basis of changes identified. Once the prototype of the changed requirements is completed, it is again handed over to the specialist for feedback and this process goes on as long as the physician gets satisfied with the prototype delivered. This process is repeated for all individual modules. After the entire prototype modules are approved, the final development is 
done for the improvement of quality measures of the system. In this way Rapid Prototype is helping in the development of a highly reliable system which is being verified by the user after each single step and the error cost is reducing very much since all the errors are being removed with the development of every next prototype.

Following steps are to be followed during the development of system:

- Requirements Elicitation

- Requirements Specifications

- Architectural Design

- Implementation

- Testing

- $\quad$ Feedback form Specialist

- Improved Prototype Systems development until approval from user

- Improvement of Quality of Final prototype

- Deployment

- Maintenance

Figure3 shows an overview of flow of development from requirements gathering to the completion and maintenance of system.

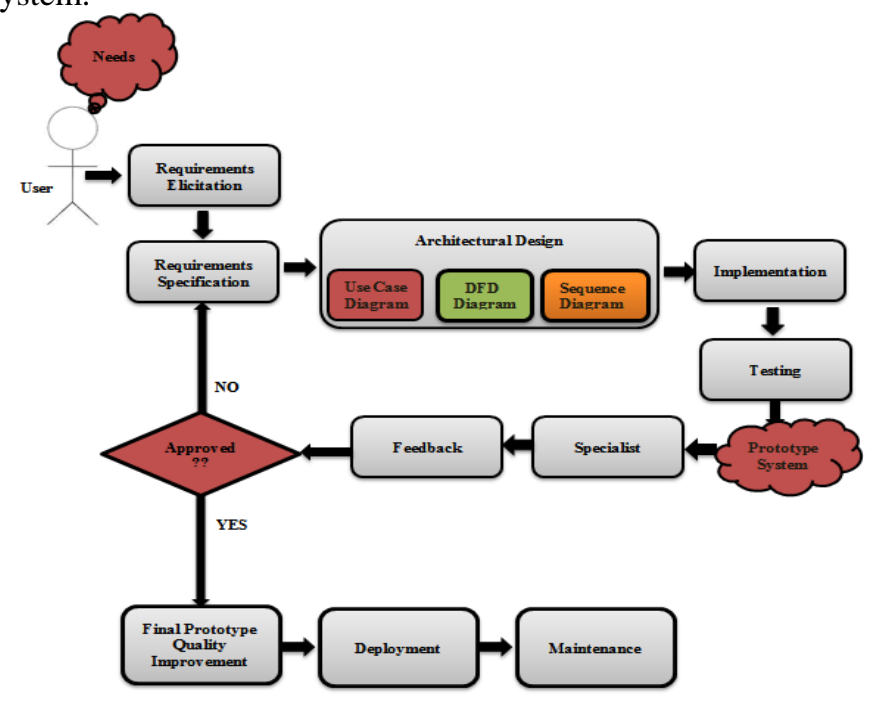

Figure 3: Flow Diagram of the Algorithm

\section{A. Requirements Elicitation}

Requirements Elicitation is the first most step of any project development. In this step maximum number of possible requirements are tried to collect. These requirements are not in a well written and structured form but this is the step where most of the information is collected about the system under development. The requirements for this project are collected from the Ophthalmological Department of AFIO. Out of multiple techniques of requirements elicitation e.g. Brainstorming, Interviewing, Questionnaires etc., the requirements for this project are collected through the Interviewing technique. Many interviews are conducted with the potential users and stakeholders of the project and maximum numbers of requirements are collected for the project. Information shown in Figure4 about the product has been collected after all the interview sessions.
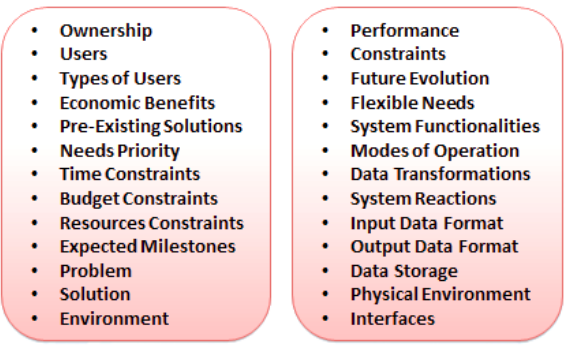

$$
\begin{aligned}
& \text { - Standards } \\
& \text { - Rpeed } \\
& \text { - Response time } \\
& \text { - Troughput } \\
& \text { - Usabing } \\
& \text { - Security } \\
& \text { - System Backup } \\
& \text { - Availability } \\
& \text { - Reliability } \\
& \text { - Maintainability } \\
& \text { - Precision } \\
& \text { - Accuracy }
\end{aligned}
$$

Figure 4: Requirements Elicitation Information Gathered

\section{B. Requirements Specification}

In requirements Specification stage requirements roughly gathered are studied deeply and their feasibility is estimated and on the basis of feasibility of each requirement three different categories are defined for a structured view of requirements shown in Figure5. Three types of requirements are:

- Functional Requirements

- Non-Functional Requirements

- Constraint Requirements
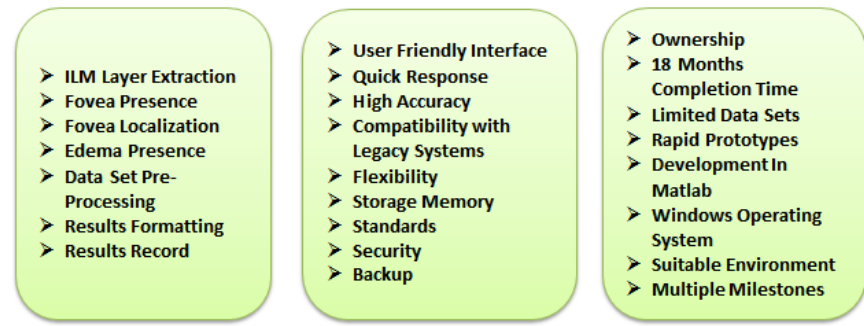

Figure 5: (Left) Functional Requirements; (Middle) NonFunctional Requirements; (Right) Constraint Requirements

\section{Architectural Design}

Architectural design consists of high level and low level design of the system. It represents the whole system as a model. The model can be in different forms like Use Case diagram, Data Flow Diagram, Sequence Diagram etc. These diagrams are used for the understanding of the system. These diagrams also help in clarifying different confusions about any functionality or any other thing. These diagrams are given to the users of the system for an overview of the system to be designed and if there is any misunderstanding then it is modified at this point. Hence saving the time, effort and resources. Following are some design diagrams used for the understanding and overview of the system:

- $\quad$ Use Case Diagram

- Data Flow Diagram

- $\quad$ Sequence Diagram

Use case diagram is helpful in understanding of the requirements as it is a form of user and system interaction and each use case depicts a scenario of interaction between the user of the system and the system. The user/actor can be a person and can also be some other system. Figure6 is a use 
case diagram showing the whole system and its interaction with users.

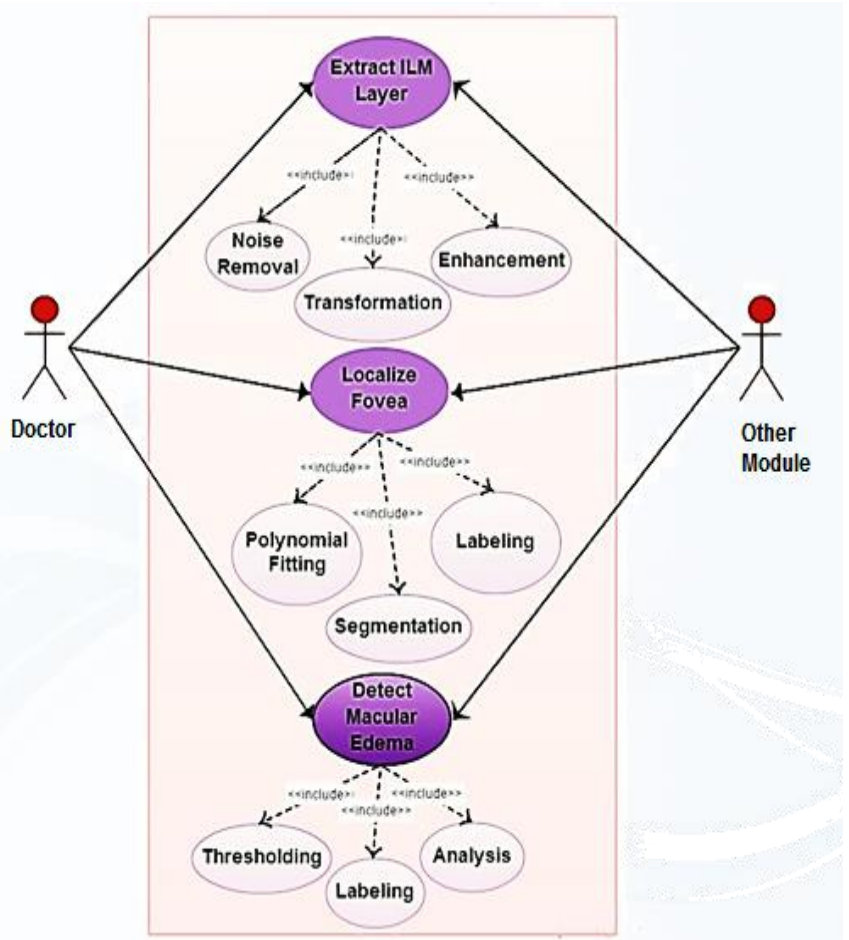

Figure 6: Edema Detection Use Case Diagram

Data flow diagrams show the flow of data between different units and processes. It helps in understanding the project in terms of data processing. It also helps in testing phase as it keeps track of the data flowing between nodes hence an error cause can be estimated. Figure7 shows the data flow diagram of the system.

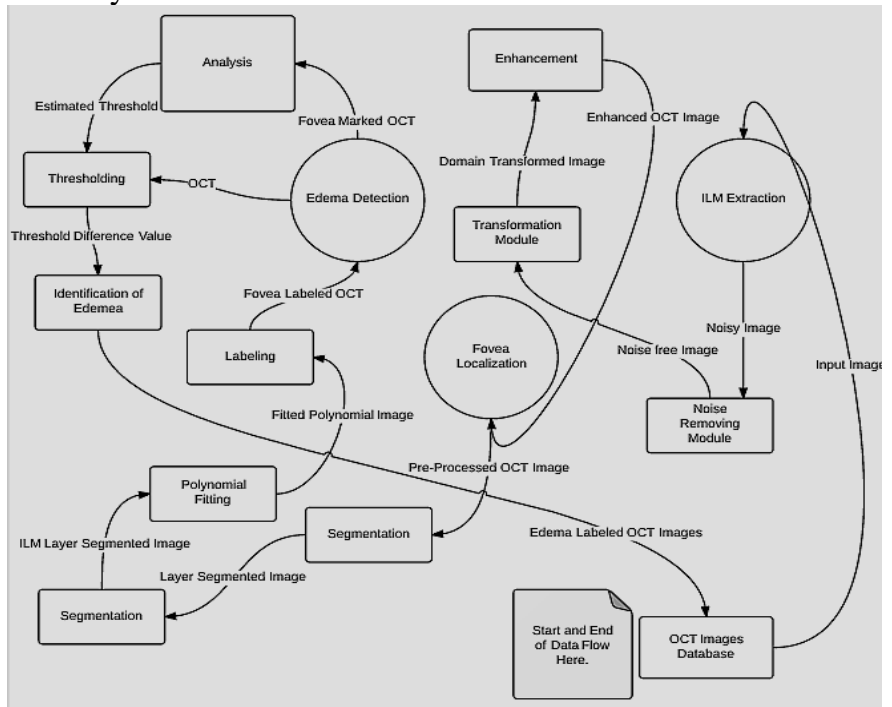

Figure 7: System Data Flow Diagram

A sequence diagram shows the occurrences of different processes at specific intervals of time from start to end of a process. It also shows the life time of different sub processes. Figure8 shows the sequence diagram of the system developed.

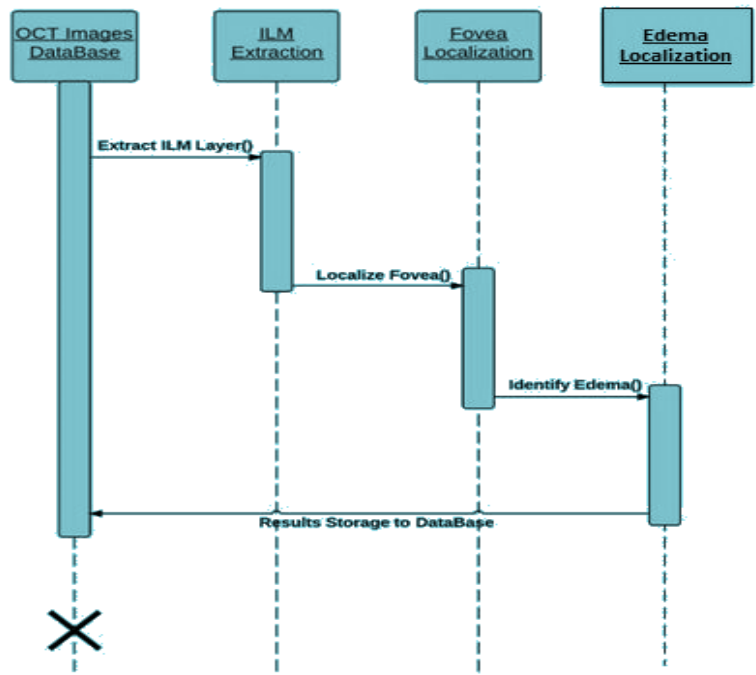

Figure 8: System Sequence Diagram

\section{Implementation, Feedback, Modifications}

The whole project is divided into three modules.
1. ILM extraction
2. Fovea Localization
3. Edema Detection

There are also sub modules of each main module. Figure9 shows the division of whole project into different modules. Since the modules are not independent but are each next module is depending upon the previous one. That's why three sub modules of main module are developed one by one. First of ILM Extraction module is developed by rapid prototyping. After 4-5 prototypes this module has been approved and finalized. After that the second module is developed by rapid prototyping. All the errors and bugs are removed with each next prototype. Similarly final module has been approved and finalized after some prototypes. Fovea localization and edema detection algorithms are changed many times as the results were either not good or were not acceptable by user. Rapid prototyping has helped in finally achieving a perfect system which is acceptable and according to the needs of user.

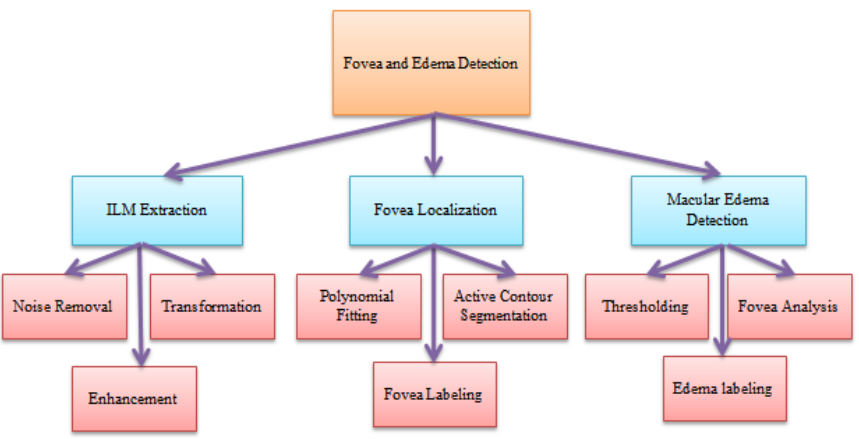

Figure 9: Modules Divisions

The main aim of the system under development is the conversion of an OCT image into a Fovea marked image and after that the labeling of that image as Edema or Non-Edema scan. Following processing is done from Input image to the 
conversion of Output image. Figure10 is an overview of Methodology steps.

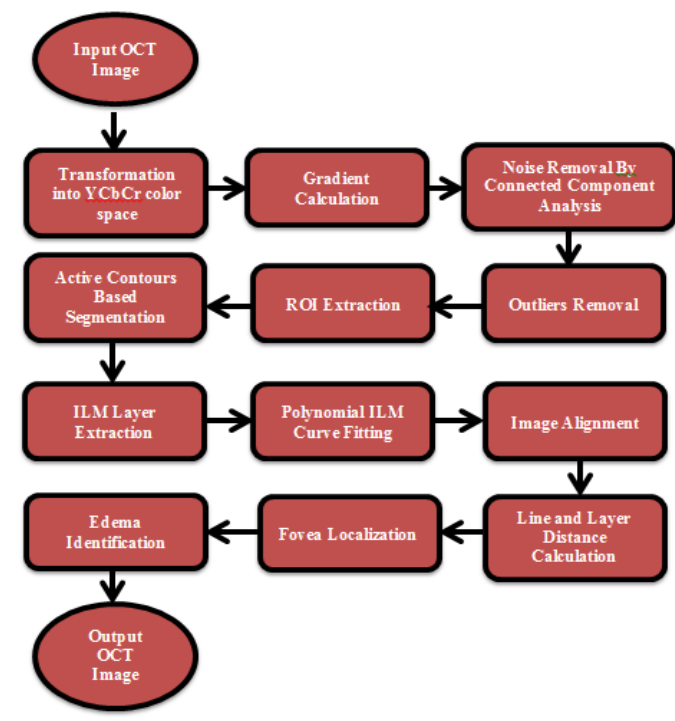

Figure 10: Implementation Overview

Since Macular edema causes prominent changes in top OCT layer of retina which contains fovea so for the purpose of Edema detection, Fovea is to be localized first and for that Extraction of region of Interest (ROI) is the main objective first. Hence an Input OCT image is taken as shown in Figure11(a). Then the input image is converted into $\mathrm{YCbCr}$ color space in Figure11(b) to get prominent layers boundaries. In the next step gradient of each color component is calculated separately using Prewitt operator as each color component contributes in the layer boundary formation. After that gradients of all three color components are combined and Thresholding is applied to remove background and much Noisy small objects are removed by performing Connected Components Analysis. Further Noise is removed by taking distance between centroids of each object and extra noisy small objects are removed on the basis of minimum threshold distance between objects. Small objects at a distance greater than threshold are removed. Figure11(c) shows the centroids of each object marked in blue and Figure11(d) shows noise free ROI extracted from original input grayscale image. This ROI is further used as an initial Mask for Active Contours Based Segmentation. This segmentation provides fine layer boundaries out of input grayscale image. Figure11(e) shows the segmented layers region. After that the upper and lower layer boundaries are separated out of segmented image and smoothing of boundaries is done by performing Polynomial Curve Fitting. Figure11(f) shows the upper and lower layer boundaries. Next the alignment of layers is done by drawing a straight line between two extreme points of layers. It helps in alignment of tilted OCT images. Since Edema is the swelling in macula which results in a Rise in macular region of OCT. Point by Point distance between upper layer and Straight line drawn is taken and the maximum distance between these two is noted. If the maximum distance value is greater than a specific threshold and lies in the middle area of layer than it is marked as an Edema otherwise as Non-edema. Figure11(g) shows the aligned and Edema Marked OCT Image.

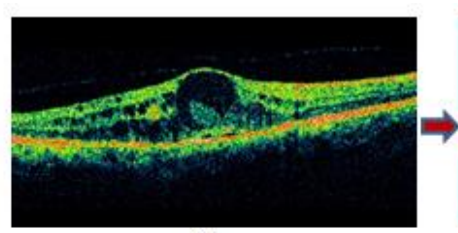

(a)

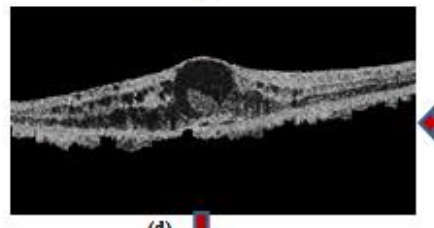

(d) 1

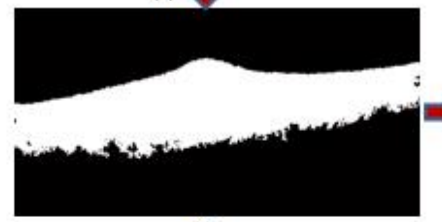

(e)

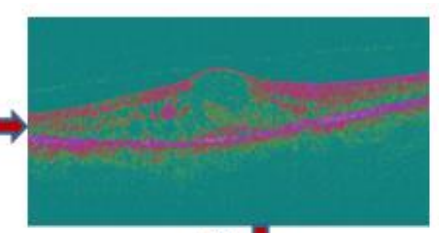

(b)
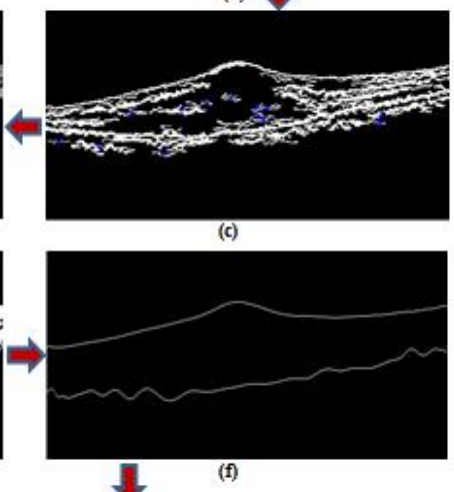

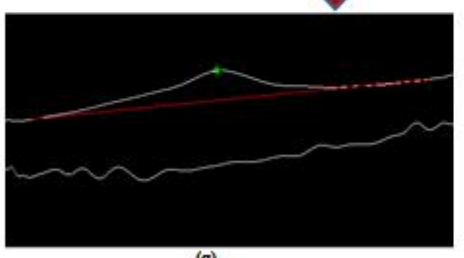

(g)

Figure 11: (a) Input Image, (b) Transformed Image, (c) Centroids Labeled, (d) ROI, (e) Segmentation, (f) Layers Boundaries, (g) Edema Labeled Output Image

\section{E. Quality Improvement, Deployment, Maintainance}

White Box testing of the system developed is performed by Statement Coverage and Boundary Value Analysis is used for Black Box testing i.e. the testing of each module and each sub module. In boundary value analysis, each functionality is tested by three input values. One is the normal Image, Second is abnormal and third one is poor quality and corrupt image. In this way all the functionalities are first unit tested for these three kind of images. After that sun modules are combined and integration testing is done. Finally System testing is done for the whole system. This testing phase is repeated after every single prototype development for the high quality and bug free system achievement. Since in many cases the Rapid prototyping model does miss some phases like testing mostly to provide the prototype quickly and making the quality of product risky but in this project testing is done after the development of each single prototype to keep the system totally error free. Also the performance testing of final product after prototyping phase completion is done by its usability testing, stress testing, Recovery Testing etc. to increase the quality of end product.

Next thing is the quality improvement of the final product which is improved in terms of response. After this the system has been deployed and the integration with legacy system is improved in the maintenance phase. 


\section{RESUlts}

Since there are no Datasets available for such system, that's why the OCT images used for the testing of Edema Identification are collected from AFIO. First the images are manually marked by some Specialist and then these images are labeled by using this system. Finally the results of both images are compared and hence the performance and accuracy of system is calculated. A local dataset of 50 images is collected for testing. The dataset contains both with Edema and without Edema presence images in which 15 images are normal and 35 are with Edema. This system correctly classifies 14 images normal and 28 images of Edema and hence the accuracy of system is $84 \%$ with 0.93 Sensitivity value and 0.8 Specificity value. Figure12 shows the results of some randomly selected images from dataset.
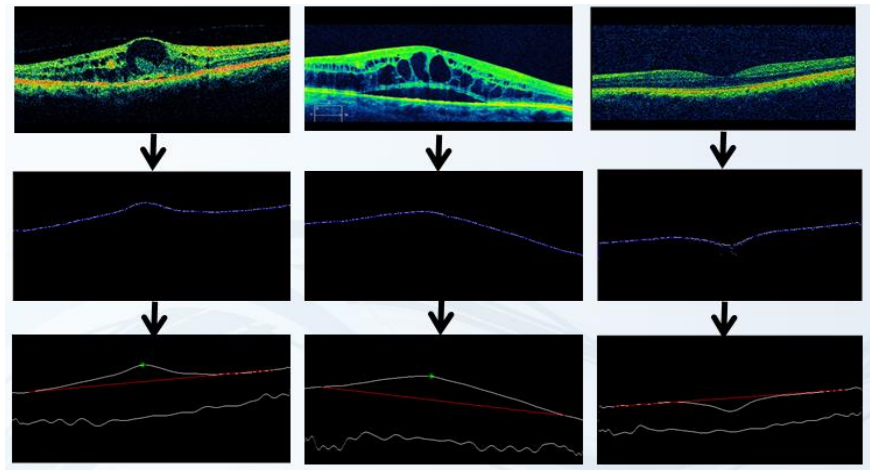

Figure 12: (Top Row) Input Images, (Middle Row) Smooth Top Layer Extracted, (Bottom Row) Edema Localized OCT Images

\section{CONCLUSION \& Discussion}

SDLC is the step by step development of a system to get an Efficient and Reliable system. It helps in Organizing and Efficiently managing the development life cycle to achieve the desired product within available Time and Resources. OCT imaging is a new technique for the detailed imaging of retina. Analysis of OCT images is helpful in detection of multiple diseases as the changes occurring in the retinal layers due to some disease can be easily observed with OCT image analysis. Macular Edema is the disease in which the sharp and pin-point vision gets affected. Since there is no work already done for the detection of such things using OCT imaging so in this research a new technique is proposed for the detection of Edema in OCT images using an appropriate SDLC model. It works on the principle of line-layer distance calculation and the labeling of Edema at the maximum positive distance point. Before that the upper most retinal layer is successfully extracted out of OCT image which is the key element for further Edema Identification processing. The extraction of ILM layer is done by using Active contour Based segmentation and polynomial Curve fitting techniques. Similarly the identification of Edema is done on the basis of rise in fovea region from a specific threshold. This system is further tested for a data set of 50 images and Edema detection gives an accuracy of $84 \%$.

\section{ACKNOWLEDGMENT}

We might want to recognize the assistance and exceptionally kind support of AFIO (Armed Forces Institute of Ophthalmology), Rawalpindi. We additionally say thanks to National ICT R\&D fund, Pakistan for their monetary support.

\section{REFERENCES}

[1] Asim, K.M., Basit, A. , Jalil, A.. "Detection and localization of fovea in human retinal fundus images," 2012 International Conference on Emerging Technologies (ICET), 2012.

[2] Guven, A. ; Oner, A.O. ; Kara, S. "Automated location of optic disk and fovea in color fundus images" $14^{\text {th }}$ National Biomedical Engineering Meeting, 2009. BIYOMUT 2009.

[3] Ziyang Liang ; Wong, D.W.K. ; Jiang Liu ; Ngan-Meng Tan ; Xiangang Cheng ; Cheung, G.C.M. ; Bhargava, M. ; Tien Yin Wong "Automatic fovea detection in retinal fundus images" Industrial Electronics and Applications (ICIEA), 2012.

[4] Veras, R., Silva, R. ; Aires, K. ; Medeiros, F. "Automatic Detection of Fovea in Retinal Images Using Fusion of Color Bands" Graphics, Patterns and Images (SIBGRAPI), 2014.

[5] Kovacs, L. ; Qureshi, R.J. ; Nagy, B. ; Harangi, B. ; Hajdu, A. "Graph based detection of optic disc and fovea in retinal images" Soft Computing Applications (SOFA), 2010.

[6] Samanta, S; Saha, S.K. ; Chanda, B. "A Simple and Fast Algorithm to Detect the Fovea Region in Fundus Retinal Image" Emerging Applications of Information Technology (EAIT), 2011.

[7] Yogesh Kumar A., Sasikala M "Texture Analysis of Retinal Layers in Spectral Domain OCT Images" International Journal of Emerging Technology and Advanced Engineering Volume 2, Issue 12, December 2012.

[8] Andrew Lang, Aaron Carass, Elias Sotirchos, Peter Calabresi, and Jerry L. Prince "Segmentation of retinal OCT images using a random forest classifier”Proc SPIE. March 13, 2013.

[9] Yang Q, Reisman CA, Wang Z, Fukuma Y, Hangai M, Yoshimura N, Tomidokoro A, Araie M, Raza AS, Hood DC, Chan K. "Automated layer segmentation of macular OCT images using dual scale gradient information". Opt Express. 2010.

[10] Garvin M, Abramoff M, Wu X, Russell S, Burns T, Sonka M. "Automated 3-D intra retinal layer segmentation of macular spectraldomain optical coherence tomography images”. IEEE Trans MedImag. 2009.

[11] Thomas Walter, Jean-Claude Klein, Pascale Massin, and Ali Erginay “A Contribution of Image Processing to the Diagnosis of Diabetic Retinopathy-Detection of Exudates in Color Fundus Images of the Human Retina" IEEE TRANSACTIONS ON MEDICAL IMAGING, VOL. 21, NO. 10, OCTOBER 2002.

[12] Umer Aftab and M. Usman Akram "Automated Identification of Exudates for Detection of Macular Edema” 2012 Cairo International Biomedical Engineering Conference (CIBEC) Cairo, Egypt, December 20-21, 2012.

[13] M. U. Akram and S. A. Khan, "Automated detection of dark and bright lesions in retinal images for early detection of diabetic retinopathy", Journal of Medical Systems (JOMS), vol. 36, no. 5, 3151-3162, 2012.

[14] A. Tariq, M. U. Akram, A. Shaukat, S. A. Khan, “Automated Detection and Grading of Diabetic Maculopathy in Digital Retinal Images", Journal of Digital Imaging, vol. 26, no. 4, pp. 803-812, 2013.

[15] M. U. Akram, A. Tariq, M. A. Anjum, M. Y. Javed, "Automated Detection of Exudates in Colored Retinal Images for Diagnosis of Diabetic Retinopathy", OSA Journal of Applied Optics, vol. 51 no. 20, 4858-4866, 2012.

[16] M. U. Akram, S. Khalid, S. A. Khan, "Identification and Classification of Microaneurysms for Early Detection of Diabetic Retinopathy", Pattern Recognition, vol. 46, no.1, 107-116, 2013. 